\title{
A New Two-Parameter Lifetime Distribution: Properties, Applications and Different Method of Estimations
}

\author{
Morad Alizadeh , Mahdi Emadi , Mahdi Doostparast \\ Department of Statistics, Ferdowsi University of Mashahd, Iran
}

\begin{abstract}
In this paper, we introduce a new two-parameter distribution which is called new Odd Log-Logistic Half-Logistic (NOLL-HL) distribution. Theoretical properties of this model including the hazard function, survival function, asymptotic, extreme value, quantile function, moments, conditional moments, mean residual life, mean past lifetime, coefficients of skewness and kurtosis, entropy and order statistics are derived and studied in details. The maximum likelihood estimates of parameters are compared with various methods of estimations by conducting a simulation study. Finally, two real data sets are illustrated the purposes.
\end{abstract}

Keywords Odd log-logistic; Half-Logistic distribution distribution; Moments; Mean residual life; Mean past lifetime; Maximum likelihood estimation.

\section{AMS 2010 subject classifications 60E05}

DOI: $10.19139 /$ soic.v7i2.653

\section{Introduction}

Modeling and analyzing lifetime data are important aspects of statistical research in many applied sciences such as engineering, medicine, economics. In many applied areas such as lifetime analysis, finance and insurance, we need extended forms of distributions. So, several methods for generating new families of distributions have been studied. Some attempts have been made to define new families of probability distributions that extend well-known families of distributions and with great flexibility in modeling data in practice.

In literature, there exist many generalized G-classes of distributions where one or more parameter(s) are added to the baseline distribution.

Gleaton and Lynch [11] defined a new transformation of distribution function called the odd log-logistic-G (OLLG) family with one additional shape parameter $\alpha>0$ by the $\mathrm{cdf}$

$$
F(x ; \alpha, \Theta)=\frac{G(x ; \Theta)^{\alpha}}{G(x ; \Theta)^{\alpha}+\bar{G}(x ; \Theta)^{\alpha}},
$$

where $G(x ; \Theta)$ is the baseline cdf $G$ with vector parameter $\Theta$ and $\bar{G}(x ; \Theta)=1-G(x ; \Theta)$. The corresponding pdf of (3) is given by

$$
f(x ; \alpha, \Theta)=\frac{\alpha g(x ; \Theta) G(x ; \Theta)^{\alpha-1} \bar{G}(x ; \Theta)^{\alpha-1}}{\left[G(x ; \Theta)^{\alpha}+\bar{G}(x ; \Theta)^{\alpha}\right]^{2}} .
$$

In general a random variable $X$ with pdf (4) is denoted by $X \sim \operatorname{OLL}-\mathrm{G}(\alpha, \Theta)$.

An interpretation of the OLL family (3) can be given as follows. Let $X$ be a random variable describing a stochastic

\footnotetext{
*Correspondence to: Mahdi Emadi (Email: emadi@um.ac.ir ). Department of Statistics, Ferdowsi University of Mashahd, Iran.
}

ISSN 2310-5070 (online) ISSN 2311-004X (print)

Copyright (C) 2019 International Academic Press 
system by the cdf $G(x)$. If the random variable $X$ represents the odds, the risk that the system following the lifetime $X$ will be not working at time $x$ is given by $y=G(x) /[1-G(x)]$. If we are interested in modeling the randomness of the odds by the log-logistic pdf $r(y)=\alpha y^{\alpha-1} /\left(1+y^{\alpha}\right)^{2}$ (for $y>0$ ), the cdf of $Y$ is given by

$$
\operatorname{Pr}(Y \leq y)=\Pi\left(\frac{G(x)}{1-G(x)}\right),
$$

where $\Pi(t)=\frac{t^{\alpha}}{1+t^{\alpha}}$ is the cdf of log-logistic distribution. Some extension of OLL-G distributions were developed by Alizadeh et.al [2] and Cordeiro et.al [9].

Let $Q_{G}($.$) denote the quantile function of G$, for generating data from this distribution, if $u \sim u(0,1)$ then

$$
X_{u}=Q_{G}\left\{\frac{u^{\frac{1}{\alpha}}}{u^{\frac{1}{\alpha}}+(1-u)^{\frac{1}{\alpha}}}\right\}
$$

has cdf (3).

Balakrishnan [4] proposed the half-logistic (HL) distribution as a lifetime increasing hazard rate function. The cdf $\mathrm{f}$ of $\mathrm{HL}$ is given by

$$
G(x)=\frac{1-\mathrm{e}^{-\mathrm{x}}}{1+\mathrm{e}^{-\mathrm{x}}}
$$

where $x>0$. The pdf of HL distribution is

$$
g(x)=\frac{2 \mathrm{e}^{-\mathrm{x}}}{\left(1+\mathrm{e}^{-\mathrm{x}}\right)^{2}}
$$

Let $r(t)$ be the probability density function (pdf) of a random variable $T \in[d, e]$ for $-\infty \leq d<e<\infty$ and let $W[G(x)]$ be a function of the cdf of a random variable $X$ such that $W[G(x)]$ satisfies the following conditions:

$$
\begin{cases}(i) & W[G(x)] \in[d, e], \\ (\text { ii }) & W[G(x)] \text { is differentiable and monotonically non-decreasing, and } \\ \text { (iii) } & W[G(x)] \rightarrow d \text { as } x \rightarrow-\infty \text { and } W[G(x)] \rightarrow d \text { as } x \rightarrow \infty .\end{cases}
$$

[3] defined the $T$ - $X$ family of distributions by

$$
F(x)=\int_{d}^{W[G(x)]} r(t) d t
$$

where $W[G(x)]$ satisfies the conditions (5). The pdf corresponding to (6) is given by

$$
f(x)=\left\{\frac{d}{d x} W[G(x)]\right\} r\{W[G(x)]\} .
$$

Some well-known families of distributions are special cases of T-X family, for example Kumaraswamy generated family by Cordeiro and de Castro [8] and Beta generated family by Jones [13].

By taking $W[G(x)]=\frac{G(x)^{\alpha}}{\bar{G}(x)^{\beta}}$ as an extension of odd ratio, $G($.$) as HL distribution and r(t)=\frac{1}{(1+t)^{2}}$ for $t>0$, we introduce a new family of distributions which called new Odd Log-Logistic Half-Logistic. The cdf and pdf of this family are given by

$$
F(x ; \alpha, \beta)=\int_{0}^{\frac{\left[\frac{1-\mathrm{e}^{-\mathrm{x}}}{1+\mathrm{e}^{-\mathrm{x}}}\right]^{\alpha}}{\left[1-\frac{1-\mathrm{e}^{-\mathrm{x}}}{1+\mathrm{e}^{-\mathrm{x}}}\right]^{\beta}}} \frac{d t}{(1+t)^{2}}=\frac{\left[\frac{1-\mathrm{e}^{-\mathrm{x}}}{1+\mathrm{e}^{-\mathrm{x}}}\right]^{\alpha}}{\left[\frac{1-\mathrm{e}^{-\mathrm{x}}}{1+\mathrm{e}^{-\mathrm{x}}}\right]^{\alpha}+\left[1-\frac{1-\mathrm{e}^{-\mathrm{x}}}{1+\mathrm{e}^{-\mathrm{x}}}\right]^{\beta}}
$$




$$
f(x ; \alpha, \beta)=\frac{\frac{2 \mathrm{e}^{-\mathrm{x}}}{\left(1+\mathrm{e}^{-\mathrm{x}}\right)^{2}}\left[\frac{1-\mathrm{e}^{-\mathrm{x}}}{1+\mathrm{e}^{-\mathrm{x}}}\right]^{\alpha-1}\left[1-\frac{1-\mathrm{e}^{-\mathrm{x}}}{1+\mathrm{e}^{-\mathrm{x}}}\right]^{\beta-1}\left[\alpha+(\beta-\alpha) \frac{1-\mathrm{e}^{-\mathrm{x}}}{1+\mathrm{e}^{-\mathrm{x}}}\right]}{\left\{\left[\frac{1-\mathrm{e}^{-\mathrm{x}}}{1+\mathrm{e}^{-\mathrm{x}}}\right]^{\alpha}+\left[1-\frac{1-\mathrm{e}^{-\mathrm{x}}}{1+\mathrm{e}^{-\mathrm{x}}}\right]^{\beta}\right\}^{2}}
$$

where $\alpha, \beta$ are two shape parameters and $x>0$.

cdf (8), is called new odd log-logistic half-logistic distribution and denoted by $X \sim \operatorname{NOLL}-\mathrm{HL}(, \alpha, \beta)$.

The hazard function (hrf) and survival function of this distribution are given by

$$
\begin{gathered}
h(x ; \alpha, \beta)=\frac{\left[\frac{1-\mathrm{e}^{-\mathrm{x}}}{1+\mathrm{e}^{-\mathrm{x}}}\right]^{\alpha-1}\left[\alpha+(\beta-\alpha) \frac{1-\mathrm{e}^{-\mathrm{x}}}{1+\mathrm{e}^{-\mathrm{x}}}\right]}{\left(1+\mathrm{e}^{-\mathrm{x}}\right)\left\{\left[\frac{1-\mathrm{e}^{-\mathrm{x}}}{1+\mathrm{e}^{-\mathrm{x}}}\right]^{\alpha}+\left[1-\frac{1-\mathrm{e}^{-\mathrm{x}}}{1+\mathrm{e}^{-\mathrm{x}}}\right]^{\beta}\right\}} \\
S(x ;, \alpha, \beta)=\frac{\left[1-\frac{1-\mathrm{e}^{-\mathrm{x}}}{1+\mathrm{e}^{-\mathrm{x}}}\right]^{\beta}}{\left[\frac{1-\mathrm{e}^{-\mathrm{x}}}{1+\mathrm{e}^{-\mathrm{x}}}\right]^{\alpha}+\left[1-\frac{1-\mathrm{e}^{-\mathrm{x}}}{1+\mathrm{e}^{-\mathrm{x}}}\right]^{\beta}}
\end{gathered}
$$

Figure 1. and Figure 2. provide the pdf and the hrf of $\operatorname{NOLL}-\operatorname{HL}(\lambda, \alpha, \beta)$ for different parameter values. These graphs show that the pdf of NOLL-HL is unimodal, right skew or almost symmetric. The hrf of NOLL-HL can be decreasing, increasing, bathtub, upside down and bathtub-upside down shape.

Figure 1. The sample curves of density function of NOLL-HL.
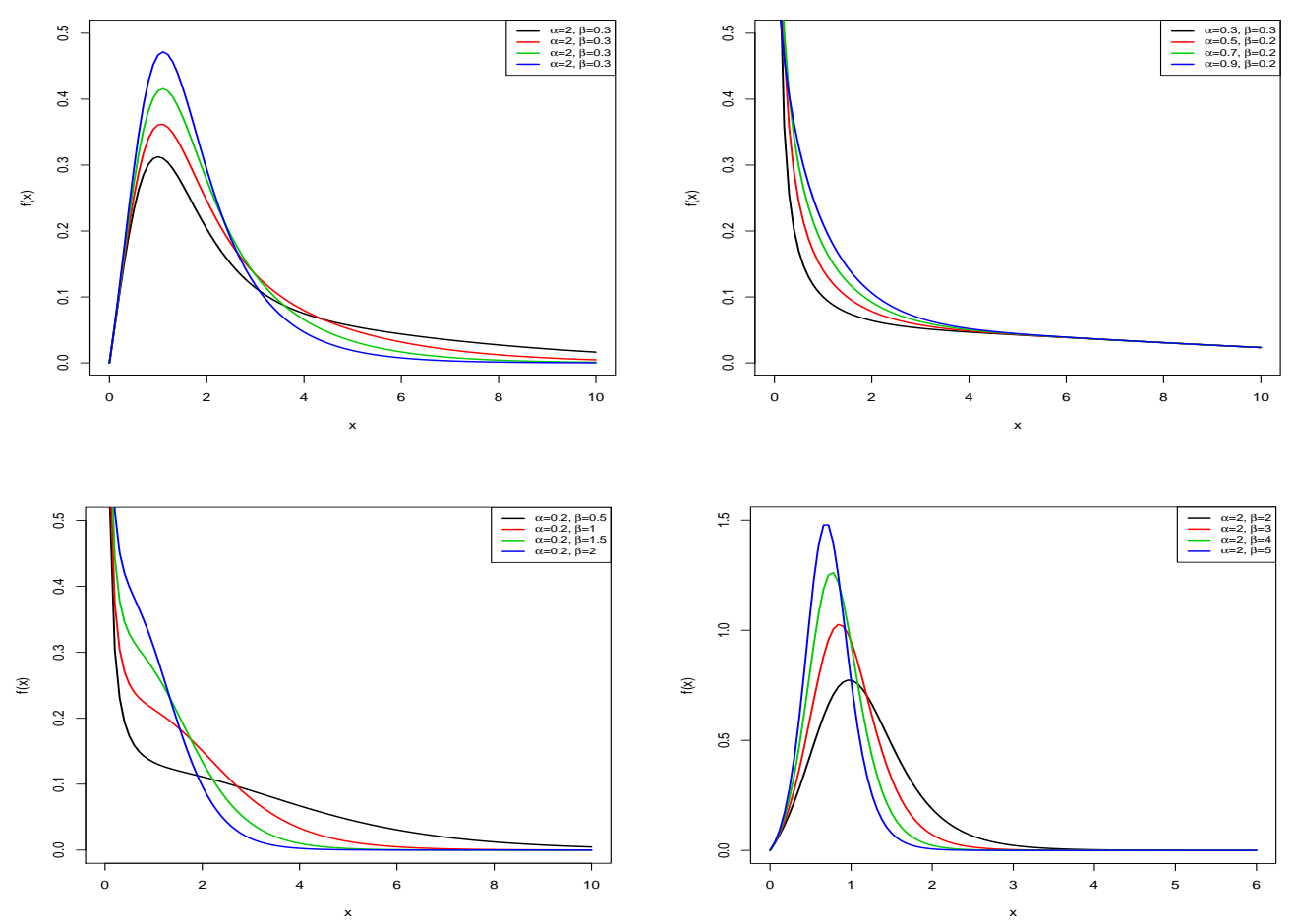

Special cases: The $N O L L-H L(\alpha, \beta)$ distribution contains as special sub-models the following well-known distributions:

For $\alpha=\beta$, we obtain Odd Log-Logistic Half-Logistic distribution (Gleaton and Lynch [11]). 
Figure 2. The sample curves of hazrd function and distribution function of NOLL-HL.
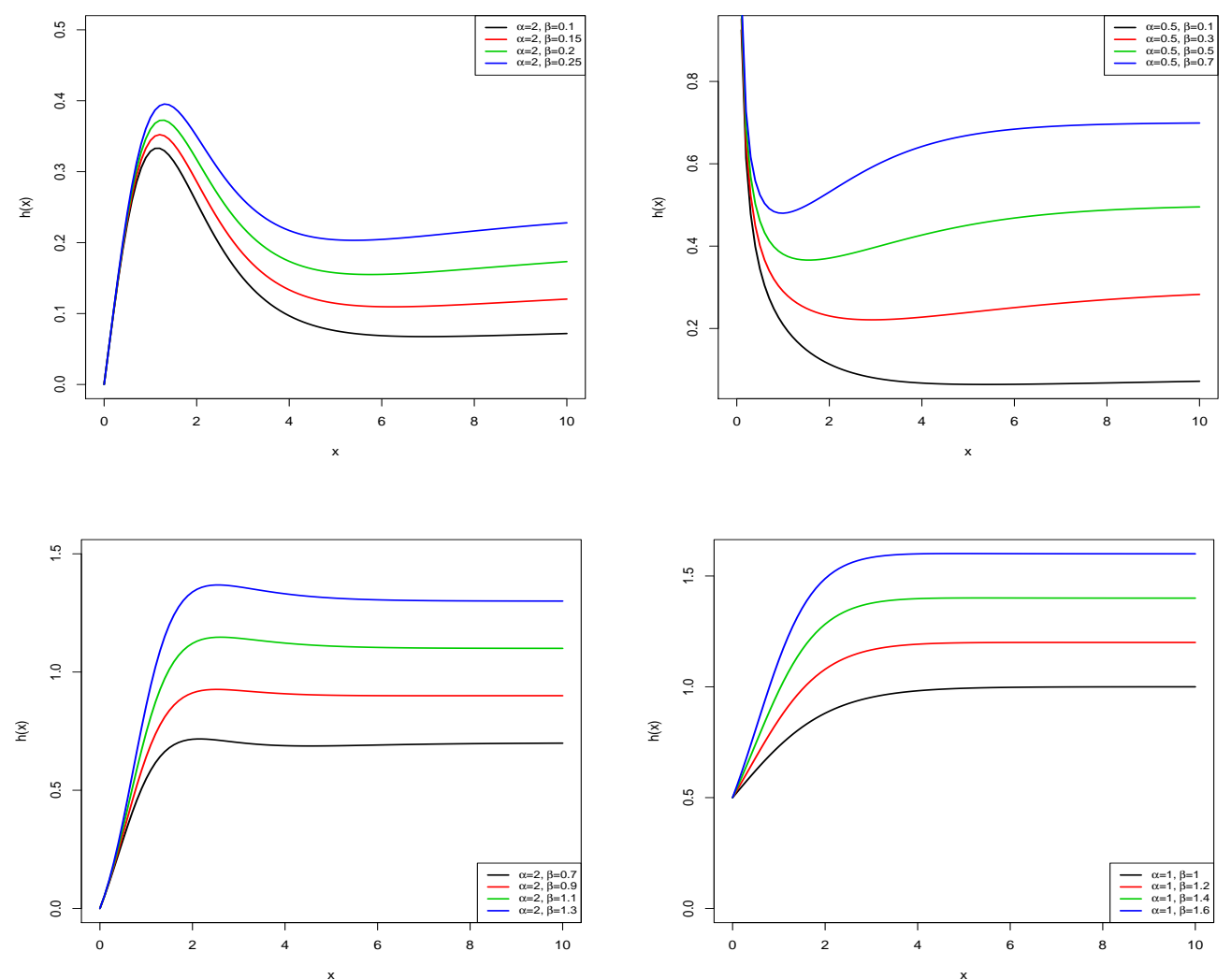

- For $\alpha=\beta=1$, we obtain Log-Logistic distribution (Balakrishnan [4]).

The paper is organized as follows: In Section 1, we construct the new family of distributions. Shape characteristics of the probability density and hazard functions of the family are investigated. Various properties of the proposed distribution are explored in Section 2. These properties include asymptotics, extreme value, mixture for cdf and pdf, moments, conditional moments, mean residual (past) lifetime, mean deviations, Lorenz and Bonferroni curves, entropies and order statistics. The method of maximum likelihood estimation of parameters is compared by five different methods of estimations with simulation study in section 3. Applications to real data sets illustrate the performance of the new family in Section 4 . In Section 5, we offer some concluding remarks.

\section{Main properties}

Some mathematical properties of the new model such as moments, moment generating function, mean residual and mean past lifetime are derived in this section. Moreover, mean deviations, Lorenz and Bonferroni curves and order statistics are presented. In the first, we investigate asymptotic properties of this model and give mixture representations for cdf and pdf. 


\subsection{Asymptotic}

The asymptotic of cdf, pdf and hrf of NOLL-HL distribution as $x \rightarrow 0$ are given by

$$
\begin{aligned}
F(x) & \sim\left(\frac{x}{2}\right)^{\alpha} & \text { as } x & \rightarrow 0, \\
f(x) & \sim \alpha\left(\frac{1}{2}\right)^{\alpha} x^{\alpha-1} & \text { as } x & \rightarrow 0, \\
h(x) & \sim \alpha\left(\frac{1}{2}\right)^{\alpha} x^{\alpha-1} & \text { as } x & \rightarrow 0 .
\end{aligned}
$$

The asymptotic of cdf, pdf, and hrf of NOLL-HL distribution as $x \rightarrow \infty$ are given as follows:

$$
\begin{aligned}
1-F(x) & \sim 2^{\beta} \mathrm{e}^{-\beta \mathrm{x}} & \text { as } \mathrm{x} \rightarrow \infty, \\
f(x) & \sim \beta 2^{\beta} \mathrm{e}^{-\beta \mathrm{x}} & \text { as } \mathrm{x} \rightarrow \infty, \\
h(x) & \sim \beta & \text { as } x \rightarrow \infty .
\end{aligned}
$$

These equations show the effect of parameters on tail of NOLL-HL distribution.

\subsection{Extreme Value}

If $\bar{X}=\left(X_{1}+\ldots+X_{n}\right) / n$ denotes the sample mean, then by the usual central limit theorem, $\sqrt{n}(\bar{X}-$ $E(X)) / \sqrt{\operatorname{Var}(X)}$ approaches the standard normal distribution as $n \rightarrow \infty$. One may be interested in the asymptotic of the extreme values $M_{n}=\max \left(X_{1}, \ldots, X_{n}\right)$ and $m_{n}=\min \left(X_{1}, \ldots, X_{n}\right)$. Let $\tau(x)=\frac{1}{\lambda}$, we obtain following equations for the cdf in (6) as

$$
\lim _{t \rightarrow 0} \frac{F(t x)}{F(t)}=\lim _{t \rightarrow 0} \frac{G(t x)^{\alpha}}{G(t)^{\alpha}}=x^{\alpha}
$$

and

$$
\lim _{t \rightarrow \infty} \frac{1-F(t+x \tau(t))}{1-F(t)}=\mathrm{e}^{-\beta x}
$$

Thus, from [15], there must be norming constants $a_{n}>0, \quad b_{n}, \quad c_{n}>0$ and $d_{n}$ such that

$$
\operatorname{Pr}\left[a_{n}\left(M_{n}-b_{n}\right) \leq x\right] \rightarrow \mathrm{e}^{-\mathrm{e}^{-\beta x}}
$$

and

$$
\operatorname{Pr}\left[c_{n}\left(m_{n}-d_{n}\right) \leq x\right] \rightarrow 1-\mathrm{e}^{-\mathrm{x}^{\alpha}}
$$

as $n \rightarrow \infty$. The form of the norming constants can also be determined. For instance, using Corollary 1.6.3 in [15], one can see that $b_{n}=F^{-1}\left(1-\frac{1}{n}\right)$ and $a_{n}=\lambda$, where $F^{-1}(\cdot)$ denotes the inverse function of $F(\cdot)$.

\subsection{Mixture representations for the cdf and pdf}

In this subsection, we provide alternative mixture representations for the pdf and cdf of $\mathrm{X}$. Using generalized binomial expansion we can obtain

$$
\left[\frac{1-\mathrm{e}^{-\mathrm{x}}}{1+\mathrm{e}^{-\mathrm{x}}}\right]^{\alpha}=\sum_{k=0}^{\infty} a_{k}\left[\frac{1-\mathrm{e}^{-\mathrm{x}}}{1+\mathrm{e}^{-\mathrm{x}}}\right]^{k}
$$


where $a_{k}=\sum_{i=k}^{\infty}(-1)^{i+k}\left(\begin{array}{c}\alpha \\ i\end{array}\right)\left(\begin{array}{c}i \\ k\end{array}\right)$ and

$$
\left[\frac{1-\mathrm{e}^{-\mathrm{x}}}{1+\mathrm{e}^{-\mathrm{x}}}\right]^{\alpha}+\left[1-\frac{1-\mathrm{e}^{-\mathrm{x}}}{1+\mathrm{e}^{-\mathrm{x}}}\right]^{\beta}=\sum_{k=0}^{\infty} b_{k}\left[\frac{1-\mathrm{e}^{-\mathrm{x}}}{1+\mathrm{e}^{-\mathrm{x}}}\right]^{k}
$$

where $b_{k}=a_{k}+(-1)^{k}\left(\begin{array}{c}\beta \\ i\end{array}\right)$.

Then we can write

$$
F(x)=\frac{\sum_{k=0}^{\infty} a_{k}\left[\frac{1-\mathrm{e}^{-\mathrm{x}}}{1+\mathrm{e}^{-\mathrm{x}}}\right]^{k}}{\sum_{k=0}^{\infty} b_{k}\left[\frac{1-\mathrm{e}^{-\mathrm{x}}}{1+\mathrm{e}^{-\mathrm{x}}}\right]^{k}}=\sum_{k=0}^{\infty} c_{k}\left[\frac{1-\mathrm{e}^{-\mathrm{x}}}{1+\mathrm{e}^{-\mathrm{x}}}\right]^{k}
$$

where $c_{0}=\frac{a_{0}}{b_{0}}$ and for $k \geq 1$,

$$
c_{k}=b_{0}^{-1}\left[a_{k}-b_{0}^{-1} \sum_{r=1}^{k} b_{r} c_{k-r}\right]
$$

We can write

$$
F(x)=\sum_{k=0}^{\infty} c_{k} F_{E H L}(x ;, k)
$$

where $F_{E H L}(x ; k)$ denotes the cdf of exponentiated Half-Logistic distribution with parameter $k$.

Now, by differentiation (18), the cdf of $\mathrm{X}$ can be expressed as

$$
f(x)=\sum_{k=0}^{\infty} c_{k+1} f_{E H L}(x ; k+1)
$$

where $f_{E H L}(x ; k+1)$ denotes the pdf of exponentiated Half-Logistic distribution with parameter $k+1$. Several properties of the NOLL-HL distribution can be obtained from pdf and cdf expansions given in (18) and (19), respectively.

\subsection{Moments}

. We hardly need to moments in any statistical analysis especially in applied work. Some of the most important features and characteristics of a distribution can be studied through moments (e.g., tendency, dispersion, skewness and kurtosis). Here, we give lemma, which will be used later.

\section{Lemma 1}

For $a_{1}, a_{2}, a_{4}>0$ and $a_{3}>-1$, let

$$
A\left(a_{1}, a_{2}, a_{3}, a_{4}\right)=\int_{0}^{\infty} \frac{x^{a_{1}} \mathrm{e}^{-\mathrm{a}_{2} \mathrm{x}}\left(1-\mathrm{e}^{-\mathrm{x}}\right)^{\mathrm{a}_{3}}}{\left(1+\mathrm{e}^{-\mathrm{x}}\right)^{\mathrm{a}_{4}}} d x
$$


Then after using some algebra, we obtain

$$
\begin{aligned}
A\left(a_{1}, a_{2}, a_{3}, a_{4}\right) & =\int_{0}^{\infty} \frac{x^{a_{1}} \mathrm{e}^{-\mathrm{a}_{2} \mathrm{x}}\left(1-\mathrm{e}^{-\mathrm{x}}\right)^{\mathrm{a}_{3}}}{\left(1+\mathrm{e}^{-\mathrm{x}}\right)^{\mathrm{a}_{4}}} d x \\
& =\sum_{i=0}^{\infty}\left(\begin{array}{c}
-a_{4} \\
i
\end{array}\right) \int_{0}^{\infty} x^{a_{1}} \mathrm{e}^{-\left(\mathrm{a}_{2}+\mathrm{i}\right) \mathrm{x}}\left(1-\mathrm{e}^{-\mathrm{x}}\right)^{\mathrm{a}_{3}} \mathrm{dx} \\
& =\sum_{i, j=0}^{\infty}(-1)^{j}\left(\begin{array}{c}
-a_{4} \\
i
\end{array}\right)\left(\begin{array}{c}
a_{3} \\
j
\end{array}\right) \int_{0}^{\infty} x^{a_{1}} \mathrm{e}^{-\left(\mathrm{a}_{2}+\mathrm{i}+\mathrm{j}\right) \mathrm{x}} \mathrm{dx} \\
& =\sum_{i, j=0}^{\infty}(-1)^{j}\left(\begin{array}{c}
-a_{4} \\
i
\end{array}\right)\left(\begin{array}{c}
a_{3} \\
j
\end{array}\right) \frac{\Gamma\left(a_{1}+1\right)}{\left(i+j+a_{2}\right)^{a_{1}+1}} .
\end{aligned}
$$

where $\Gamma($.$) is the gamma function.$

Next, the $n$-th moment of the NOLL-HL distribution is given as follows:

$$
E\left(X^{n}\right)=2 \sum_{k=0}^{\infty}(k+1) c_{k+1} A(n, 1, k-1, k+1) .
$$

For integer values of $n$, Let $\mu_{n}^{\prime}=E\left(X^{n}\right)$ and $\mu=\mu_{1}^{\prime}=E(X)$, then one can also find the $n$-th central moment of the NOLL-HL distribution as

$$
\mu_{n}=E(X-\mu)^{n}=\sum_{i=0}^{n}\left(\begin{array}{c}
n \\
i
\end{array}\right) \mu_{i}^{\prime}(-\mu)^{n-i}
$$

Using (21), the measures of skewness and kurtosis of the NOLL-HL distribution can be obtained as

$$
\operatorname{Skewness}(X)=\frac{\mu_{3}^{\prime}-3 \mu_{2}^{\prime} \mu_{1}^{\prime}+2 \mu_{1}^{\prime 3}}{\left(\mu_{2}^{\prime}-\mu_{1}^{\prime 2}\right)^{\frac{3}{2}}}
$$

and

$$
\operatorname{Kurtosis}(X)=\frac{\mu_{4}^{\prime}-4 \mu_{1}^{\prime} \mu_{3}^{\prime}+6 \mu_{1}^{\prime 2} \mu_{3}^{\prime}-3 \mu_{1}^{\prime 4}}{\mu_{2}^{\prime}-\left(\mu_{1}^{\prime}\right)^{2}},
$$

respectively. Additionally, the moment generating function of NOLL-HL distribution can be written as

$$
E\left[e^{t X}\right]=2 \sum_{k=0}^{\infty}(k+1) c_{k+1} A(0,1-t, k-1, k+1) .
$$

Figure 3 shows the behaviour of skewness and kurtosis of NOLL-HL distribution.

\subsection{Conditional moments:}

Here, we intend to determine the conditional moments of the new family. Let

$$
B\left(a_{1}, a_{2}, a_{3}, a_{4}, y\right)=\int_{0}^{y} \frac{x^{a_{1}} \mathrm{e}^{-\mathrm{a}_{2} \mathrm{x}}\left(1-\mathrm{e}^{-\mathrm{x}}\right)^{\mathrm{a}_{3}}}{\left(1+\mathrm{e}^{-\mathrm{x}}\right)^{\mathrm{a}_{4}}} d x .
$$

For $a_{1}, a_{2}, a_{4}>0$ and $a_{3}>-1$. Then, we obtain

$$
B\left(a_{1}, a_{2}, a_{3}, a_{4}, y\right)=\sum_{i, j=0}^{\infty}(-1)^{j}\left(\begin{array}{c}
-a_{4} \\
i
\end{array}\right)\left(\begin{array}{c}
a_{3} \\
j
\end{array}\right) \frac{\gamma\left(y\left(a_{2}+i+j\right), a_{1}+1\right)}{\left(i+j+a_{2}\right)^{a_{1}+1}}
$$


Figure 3. Skewness and Kurtosis for NOLL-HL.
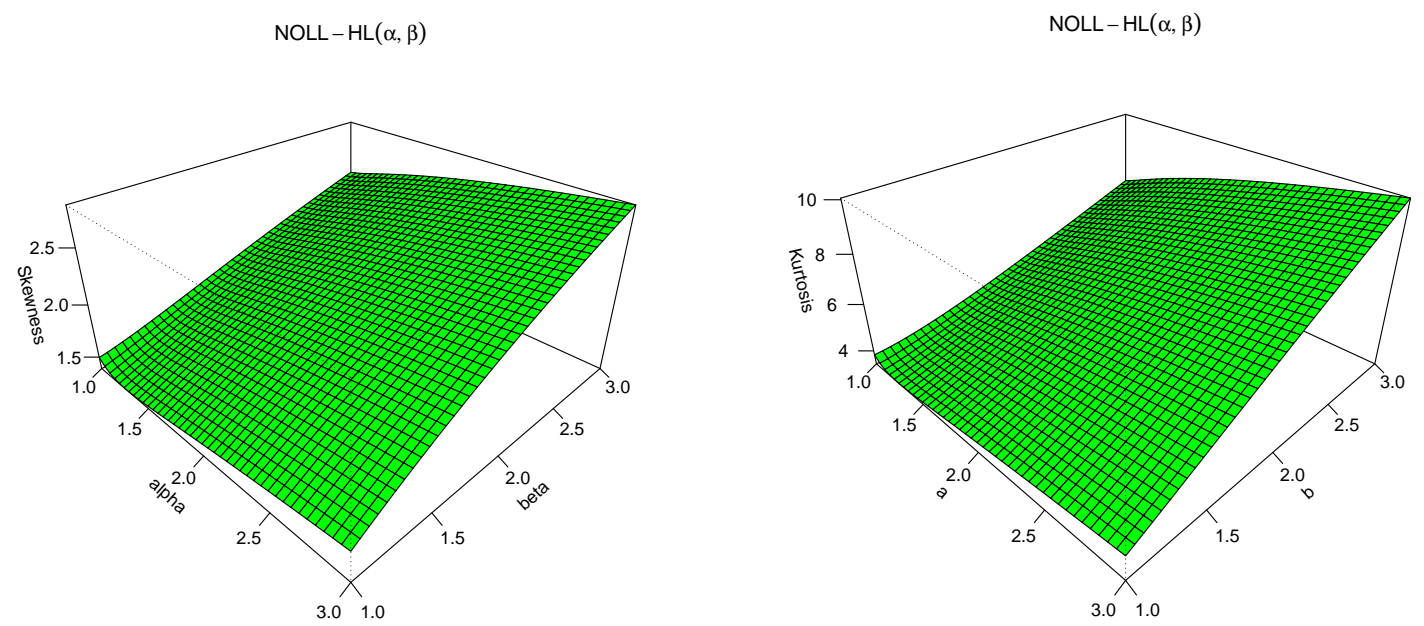

where $\gamma(z, a)=\int_{0}^{z} t^{a-1} \mathrm{e}^{-\mathrm{t}} \mathrm{dt}$ is the incomplete gamma function. So, the $n$-th conditional moments of $X$ can be expressed as

$$
E\left(X^{n} \mid X>x\right)=\frac{2}{1-F(x)} \sum_{k=0}^{\infty}(k+1) c_{k+1}[A(n, 1, k-1, k+1)-B(n, 1, k-1, k+1 ; x)]
$$

Therefore

$$
E\left(X^{n} \mid X \leq x\right)=\frac{2}{F(x)} \sum_{k=0}^{\infty}(k+1) c_{k+1} B(n, 1, k-1, k+1 ; x) .
$$

\subsection{Mean residual life}

In life testing situations, the expected additional lifetime given that a component has survived until time $x$ is a function of $x$, called the mean residual life. More specifically, if the random variable $X$ represents the life of a component, then the mean residual life is given by $M_{X}(x)=E(X-x \mid X>x)$ and can be expressed as

$$
M_{X}(x)=\mu-x-\frac{2}{1-F(x)} \sum_{k=0}^{\infty}(k+1) c_{k+1} B(1,1, k-1, k+1 ; x) .
$$

\subsection{Mean past lifetime}

In many realistic situations, the random variables are not necessarily related only to the future, but they can also refer to the past. In fact, in many reliability problems, it is of interest to consider variables of the kind $(x-X \mid X \leq x)$ for fixed $x$, called the past lifetime, which denotes the time elapsed after failure till time $x$ given that the unit has already failed by time $x$ defined for a nonnegative random variable $X$. The mean past lifetime of nonnegative random variable $\mathrm{X}$ is defined as $m_{X}(x)=E(x-X \mid X \leq x)$ and is equal to

$$
m_{X}(x)=x-\frac{2}{F(x)} \sum_{k=0}^{\infty}(k+1) c_{k+1} B(1,1, k-1, k+1 ; x) .
$$




\subsection{Mean deviations}

The mean deviations can be used as a measure of spread in a population. The mean deviations about the mean and about the median are given by

$$
\delta_{1}(X)=\int_{0}^{\infty}|x-\mu| f(x) d x
$$

and

$$
\delta_{2}(X)=\int_{0}^{\infty}|x-m| f(x) d x
$$

respectively, where $\mu=E(X)$ and $m=\operatorname{median}(X)$. These quantities can be calculated as

$$
\delta_{1}(X)=2 \mu F(\mu)-2 \int_{0}^{\mu} x f(x) d x
$$

and

$$
\delta_{2}(X)=\mu-2 \int_{0}^{m} x f(x) d x
$$

Using (18) and (26), it follows that

$$
\delta_{1}(X)=2 \mu F(\mu)-4 \sum_{k=0}^{\infty}(k+1) c_{k+1} B(1,1, k-1, k+1 ; \mu)
$$

and

$$
\delta_{2}(X)=\mu-4 \sum_{k=0}^{\infty}(k+1) c_{k+1} B(1,1, k-1, k+1 ; m) .
$$

\subsection{Bonferroni and Lorenz curves}

We can construct Bonferroni and Lorenz curves, which are important in several fields such as economics, reliability, demography, insurance and medicine. They are defined as the following:

$$
B(F(x))=\frac{1}{\mu F(x)} \int_{0}^{x} t f(t) d t
$$

and

$$
L(F(x))=\frac{1}{\mu} \int_{0}^{x} t f(t) d t
$$

By (26), (27) and (28) one can obtain

$$
B(F(x))=\frac{4}{\mu F(x)} \sum_{k=0}^{\infty}(k+1) c_{k+1} B(1,1, k-1, k+1 ; x),
$$

and

$$
L(F(x))=\frac{4}{\mu} \sum_{k=0}^{\infty}(k+1) c_{k+1} B(1,1, k-1, k+1 ; x) .
$$




\subsection{Entropies}

An entropy is a measure of variation or uncertainty of a random variable $X$. Two popular entropy measures are the Rényi and Shannon entropies (Shannon [21]; Rényi [19]). The Rényi entropy of a random variable with pdf $f(x)$ is defined by

$$
I_{R}(\gamma)=\frac{1}{1-\gamma} \log \left(\int_{0}^{\infty} f^{\gamma}(x) d x\right)
$$

for $\gamma>0$ and $\gamma \neq 1$. The Shannon entropy of a random variable $X$ is defined by $E\{-\log [f(X)]\}$. It is the special case of the Rényi entropy when $\gamma \uparrow 1$. For the Rényi entropy, after some algebraic developments, we obtain an alternative expression

$$
\begin{aligned}
f(x)^{\gamma} & =\frac{g(x)^{\gamma} G(x)^{\gamma(\alpha-1)} \bar{G}(x)^{\gamma(\beta-1)}[\alpha+(\beta-\alpha) G(x)]^{\gamma}}{\left[G(x)^{\alpha}+\bar{G}(x)^{\beta}\right]^{2 \gamma}} \\
& =g(x)^{\gamma} \sum_{i, j=0}^{\infty}(-1)^{i}\left(\begin{array}{c}
\gamma(\beta-1) \\
i
\end{array}\right)\left(\begin{array}{c}
\gamma \\
j
\end{array}\right) \alpha^{j}(\beta-\alpha)^{\gamma-j} \frac{G(x)^{\gamma(\alpha-1)+i+j}}{\left[G(x)^{\alpha}+\bar{G}(x)^{\beta}\right]^{2 \gamma}}
\end{aligned}
$$

but

$$
\frac{G(x)^{\gamma(\alpha-1)+i+j}}{\left[G(x)^{\alpha}+\bar{G}(x)^{\beta}\right]^{2 \gamma}}=\frac{\sum_{k=0}^{\infty} a_{k}^{*} G(x)^{k}}{\sum_{k=0}^{\infty} b_{k}^{*} G(x)^{k}}=\sum_{k=0}^{\infty} c_{k}^{*} G(x)^{k}
$$

where

$$
\begin{aligned}
& a_{k}^{*}=s_{k}(\gamma(\alpha-1)+i+j) \\
& b_{k}^{*}=h_{k}(\alpha, \beta ; \gamma) \text { defined in Appendix B } \\
& c_{0}^{*}=\frac{a_{0}^{*}}{b_{0}^{*}} \text { and for } k \geq 1, c_{k}^{*}=\frac{1}{b_{0}^{*}}\left[a_{k}^{*}-\frac{1}{b_{0}^{*}} \sum_{r=1}^{k} b_{r}^{*} c_{k-r}^{*}\right]
\end{aligned}
$$

Then

$$
f(x)^{\gamma}=\sum_{i, j, k=0}^{\infty} w_{i, j, k}^{\prime} g(x)^{\gamma} G(x)^{k}
$$

where

$$
w_{i, j, k}^{\prime}=(-1)^{i}\left(\begin{array}{c}
\gamma(\beta-1) \\
i
\end{array}\right)\left(\begin{array}{l}
\gamma \\
j
\end{array}\right) \alpha^{j}(\beta-\alpha)^{\gamma-j} c_{k}^{*}
$$

Then

$$
I_{\gamma}(x)=\frac{1}{1-\gamma} \log \left[\sum_{i, j, k=0}^{\infty} w_{i, j, k}^{\prime} \int_{0}^{\infty} g(x)^{\gamma} G(x)^{k}\right]
$$

The last integral can obtained by

$$
\int_{0}^{\infty} g(x)^{\gamma} G(x)^{k}=2 \sum_{l=0}^{\infty} \sum_{s=0}^{k} \frac{\left(\begin{array}{c}
-2 \gamma-k \\
l
\end{array}\right)\left(\begin{array}{l}
k \\
s
\end{array}\right)}{\gamma+s+l}
$$




\subsection{Order statistics}

Order statistics are among the most fundamental tools in nonparametric statistics and inference. Suppose $X_{1}, X_{2}, \cdots, X_{n}$ is a random sample from (8). Let $X_{1: n} \leq X_{2: n} \leq \cdots \leq X_{n: n}$ denote the corresponding order statistics. It is well known that the pdf of the $i$-th order statistic, is given by

$$
\begin{aligned}
f_{i: n}(x) & =\frac{1}{B(i, n-i+1)} F^{i-1}(x)(1-F(x))^{n-i} f(x) \\
& =\frac{1}{B(i, n-i+1)} \sum_{j=0}^{n-i}(-1)^{j}\left(\begin{array}{c}
n-i \\
j
\end{array}\right) F^{j+i-1}(x) f(x)
\end{aligned}
$$

where $B(i, n-i+1)$ is the Beta function. Here and henceforth, we use an equation by Gradshteyn and Ryzhik [12], page 17, for a power series raised to a positive integer $n$

$$
\left(\sum_{i=0}^{\infty} a_{i} u^{i}\right)^{n}=\sum_{i=0}^{\infty} d_{n, i} u^{i}
$$

where the coefficients $d_{n, i}$ (for $i=1,2, \cdots$ ) are determined from the recurrence equation (with $d_{n, 0}=a_{0}^{n}$ )

$$
d_{n, i}=\left(i a_{0}\right)^{-1} \sum_{m=1}^{i}[m(n+1)-i] a_{m} d_{n, i-m} .
$$

From Eq. (32), we can show that the density function of the $i$-th order statistic of any EE-C distribution can be expressed as

$$
f_{i: n}(x)=\sum_{r, k=0}^{\infty} e_{r, k} f_{E H L}(x ;, r+k+1),
$$

where $f_{E H L}(x ; r+k+1)$ denotes the density function of EHL distribution with parameter $r+k+1$ and

$$
e_{r, k}=\frac{n !(r+1)(i-1) ! c_{r+1}}{(r+k+1)} \sum_{j=0}^{n-i} \frac{(-1)^{j} f_{j+i-1, k}}{(n-i-j) ! j !} .
$$

Here the quantities $f_{j+i-1, k}$ can be determined given that $f_{j+i-1,0}=c_{0}^{j+i-1}$ and recursively we have

$$
f_{j+i-1, k}=\left(k c_{0}\right)^{-1} \sum_{m=1}^{k}[m(j+i)-k] c_{m} f_{j+i-1, k-m}, k \geq 1,
$$

and $c_{r}$ is given by (17). The $m$-th moments of $X_{i: n}$ is equal to

$$
E\left(X_{i: n}^{m}\right)=2 \sum_{r, k=0}^{\infty} e_{r, k} A(m, 1, r+k, r+k+2) .
$$

\section{Maximum-likelihood estimation}

We determine the maximum likelihood estimates (MLEs) of the parameters of the NOLL-HL distribution from complete samples only. Let $x_{1}, \ldots, x_{n}$ be a random sample of size $n$ from the NOLL-HL $(\alpha, \beta)$ distribution. The 
$\log$-likelihood function for the vector of parameters $\Theta=(\alpha, \beta)^{T}$ can be written as

$$
\begin{aligned}
l(\Theta) & =n \log (2)-\lambda \sum_{i=1}^{n} x_{i}-2 \sum_{i=1}^{n} \log \left(1+\mathrm{e}^{-\mathrm{x}_{\mathrm{i}}}\right) \\
& +(\alpha-1) \sum_{i=1}^{n} \log \left(q_{i}\right)+\sum_{i=1}^{n} \log \left[\alpha+(\beta-\alpha) q_{i}\right]-2 \sum_{i=1}^{n} \log \left[q_{i}^{\alpha}+\left(1-q_{i}\right)^{\beta}\right]
\end{aligned}
$$

where $q_{i}=\frac{1-\mathrm{e}^{-\mathrm{x}_{\mathrm{i}}}}{1+\mathrm{e}^{-\mathrm{x}_{\mathrm{i}}}}$ is a transformed observation.

The log-likelihood can be maximized either directly by using the SAS (Procedure NLMixed) or by solving the nonlinear likelihood equations obtained by differentiating (33). The components of the score vector $U(\Theta)$ are given by

$$
U_{\alpha}(\Theta)=\sum_{i=1}^{n} \log \left(q_{i}\right)+\sum_{i=1}^{n} \frac{1-q_{i}}{\alpha+(\beta-\alpha) q_{i}}-2 \sum_{i=1}^{n} \frac{q_{i}^{\alpha} \log \left(q_{i}\right)}{q_{i}^{\alpha}+\left(1-q_{i}\right)^{\beta}}
$$

and

$$
U_{\beta}(\Theta)=\sum_{i=1}^{n} \frac{q_{i}}{\alpha+(\beta-\alpha) q_{i}}-2 \sum_{i=1}^{n} \frac{\left(1-q_{i}\right)^{\beta} \log \left(1-q_{i}\right)}{q_{i}^{\alpha}+\left(1-q_{i}\right)^{\beta}} .
$$

\subsection{The other estimation methods}

There are several approaches to estimate the parameters of distributions that each of them has its characteristic features and benefits. In this subsection five of those methods are briefly introduced and will be numerically investigated in the simulation study. A useful summary of these methods can be seen in Dey et al. [10]. Here $\left\{t_{i} ; i=1,2, \ldots, n\right\}$ and $\left\{t_{i: n} ; i=1,2, \ldots, n\right\}$ is the random sample and associated order statistics and $F$ is the distribution function of NOLL-HL distribution.

The Least Squares (LSE) and weighted Least Squares Estimators (WLSE) are introduced by Swain et al., [23]. The LSE's and WLSE's are obtained by minimizing the following functions:

$$
S_{\mathrm{LSE}}(\alpha, \beta)=\sum_{i=1}^{n}\left(F\left(t_{i: n} ; \alpha, \beta\right)-\frac{i}{n+1}\right)^{2}
$$

and

$$
S_{\mathrm{WLSE}}(\alpha, \beta)=\sum_{i=1}^{n} \frac{(n+1)^{2}(n+2)}{i(n-i+1)}\left(F\left(t_{i: n} ; \alpha, \beta\right)-\frac{i}{n+1}\right)^{2} .
$$

Cramér-von-Mises Estimator (CME) is introduced by Choi and Bulgren [7]. The CMEs is obtained by minimizing the following function:

$$
S_{\mathrm{CME}}(\alpha, \beta)=\frac{1}{12 n}+\sum_{i=1}^{n}\left(F\left(t_{i: n} ; \alpha, \beta\right)-\frac{2 i-1}{2 n}\right)^{2} .
$$

The Anderson Darling (ADE) and Right-Tailed Anderson Darling Estimators (RTADE) are introduced by Anderson and Darling [1]. The ADE's and RTADE's are obtained by minimizing the following functions:

$$
S_{\mathrm{ADE}}(\alpha, \beta)=-n-\frac{1}{n} \sum_{i=1}^{n}(2 i-1)\left\{\log F\left(t_{i} ; \alpha, \beta\right)+\log \bar{F}\left(t_{n+1-i} ; \alpha, \beta\right)\right\}
$$

and

$$
S_{\mathrm{RTADE}}(\alpha, \beta)=\frac{n}{2}-2 \sum_{i=1}^{n} F\left(t_{i} ; \alpha, \beta\right)-\frac{1}{n} \sum_{i=1}^{n}(2 i-1) \log \bar{F}\left(t_{n+1-i} ; \alpha, \beta\right),
$$


where $\bar{F}(\cdot)=1-F(\cdot)$. Cheng and Amin [5],[6] introduced the maximum product of spacings (MPS) method as an alternative to MLEs for estimating parameters of continuous univariate distributions. The MPS's are obtained by maximizing the following functions:

$$
G(\alpha, \beta)=\left[\prod_{i=1}^{n+1} D_{i}(\alpha, \beta)\right]^{\frac{1}{n+1}}
$$

where $D_{i}(\alpha, \beta)=F\left(t_{i: n} ; \alpha, \beta\right)-F\left(t_{i-1: n} ; \alpha, \beta\right), i=1, \ldots, n, F\left(t_{0: n} ; \alpha, \beta\right)=0$ and $F\left(t_{n+1: n} ; \alpha, \beta\right)=1$.

\subsection{Simulation study}

In order to explore the estimators introduced above we consider the one model that have been used in this section , and investigate MSE of those estimators for different samples. For instance according to what has been mentioned above, for $(\alpha, \beta)=(0.9,0.6),(2,1),(3.1,0.4)$.

The performance of each method of parameters estimations for the NOLL-HL distribution with respect to sample size $\mathrm{n}$ is considered. For this aim, simulation study is done based on the following steps:

1. generate one thousand samples of size $\mathrm{n}$ from (5). This work is done simply by quantile function and generated data from uniform distribution .

2. compute the estimates for the one thousand samples, say $\left(\hat{\alpha}_{i}, \hat{\beta}_{i}\right)$ for $i=1,2, \ldots, 10000$.

3. compute the biases and mean squared errors given by

$$
\operatorname{Bias}_{\varepsilon}(n)=\frac{1}{10000} \sum_{i=1}^{10000}\left(\hat{\varepsilon}_{i}-\varepsilon\right)
$$

and

$$
M S E_{\varepsilon}(n)=\frac{1}{10000} \sum_{i=1}^{10000}\left(\hat{\varepsilon}_{i}-\varepsilon\right)^{2}
$$

for $\varepsilon=\alpha, \beta$.

We repeated these steps for $n=30,40,50, \cdots 500$ with mentioned special case of parameters. Then we computed $\operatorname{bias}_{\varepsilon}(n)$ and $M S E_{\varepsilon}(n)$ for $\varepsilon=\alpha, \beta$ and $n=30,40, \cdots 500$. To obtain the value of the estimators, we have used the optima function and Nelder-Mead method in R. The result of the simulations of this subsection is shown in Figures 4 to 6 .
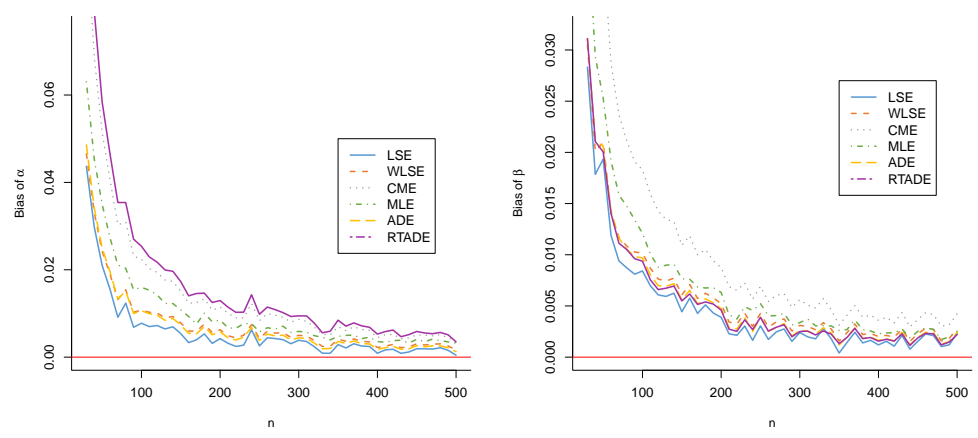

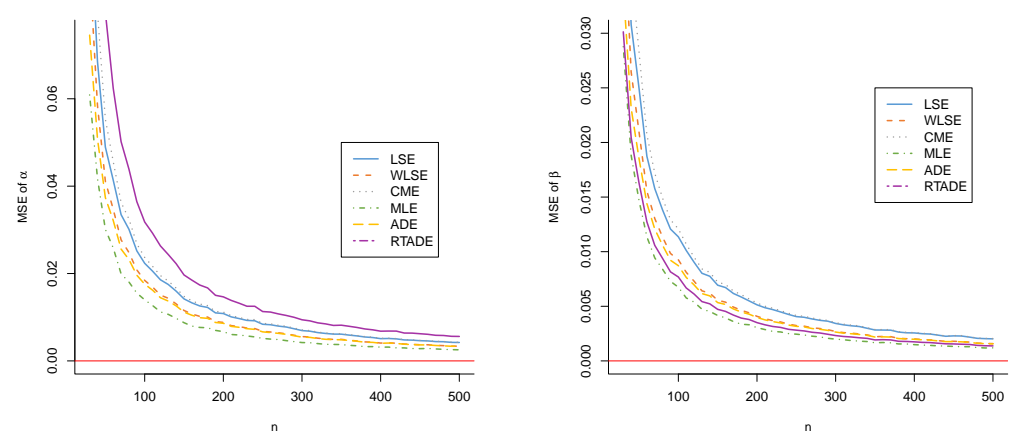

Figure 4. Bias of estimations for parameter values $(\alpha, \beta)=(0.9,0.6)$
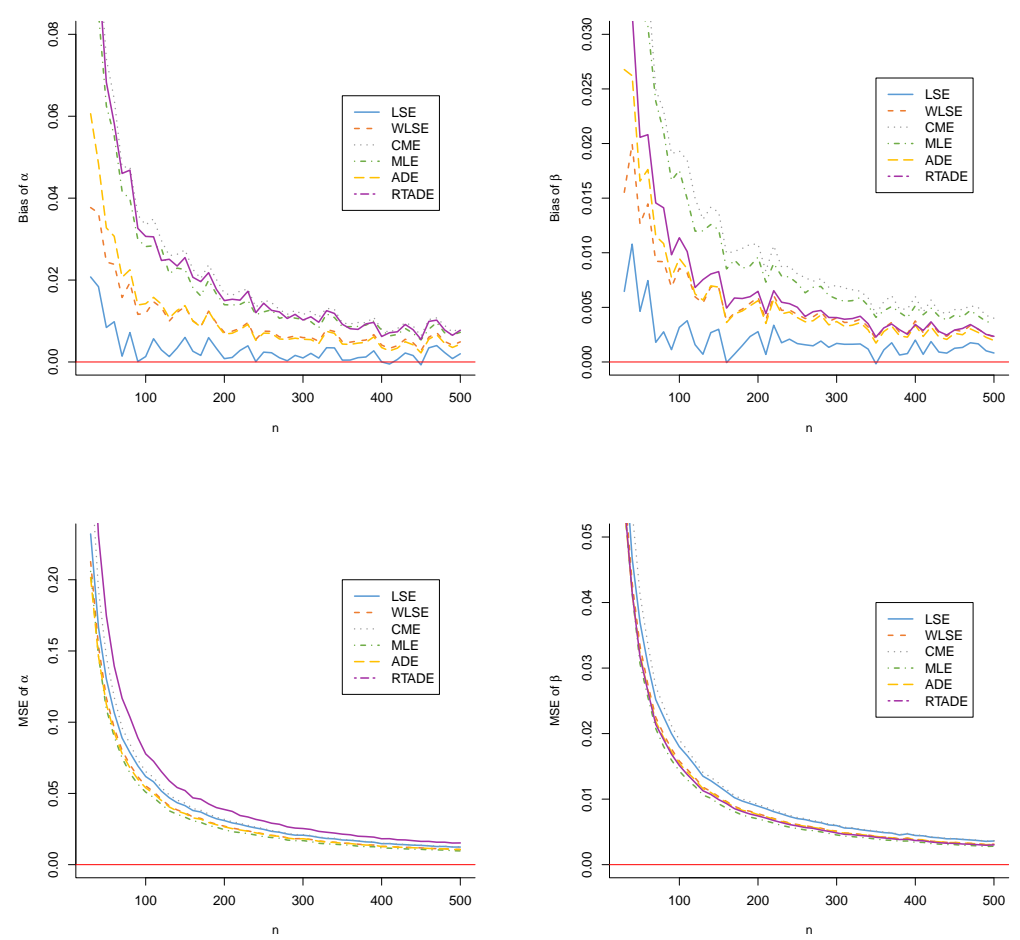

Figure 5. Bias of estimations for parameter values $(\alpha, \beta)=(2,1)$

A general result about above figures is that MSE plot for two parameters with the increase in the volume of the sample all methods will approach to zero and this verifies the validity of the these estimation methods and numerical calculations for the distribution parameters NOLL-HL.

Some of other results are mentioned as follow:

- For estimating $\alpha$, LSE method has the minimum amonut of bias.

- For estimating $\beta$, LSE method has the minimum amonut of bias.

- For estimating $\alpha$, MLE method has the minimum amonut of MSE.

- For estimating $\beta$, MLE method has the minimum amonut of MSE. 

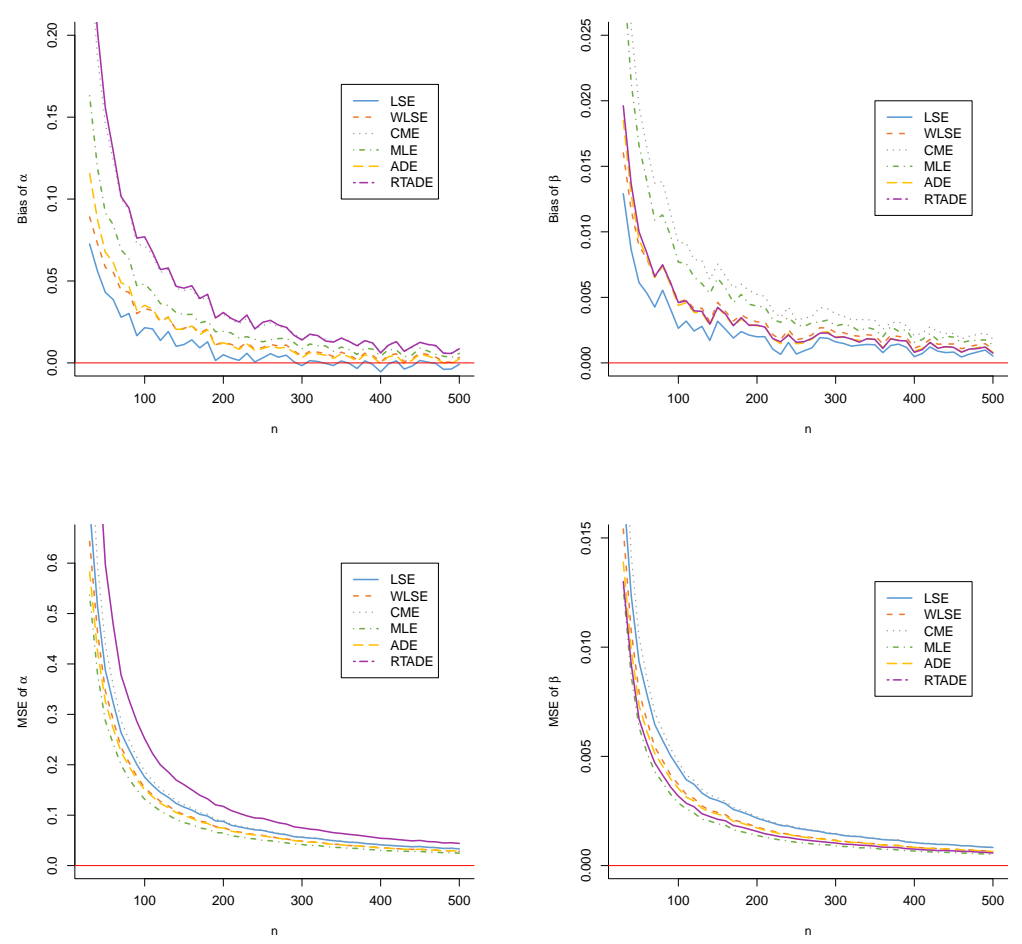

Figure 6. Bias of estimations for parameter values $(\alpha, \beta)=(3.1,0.4)$

\section{Applications}

In this section, we present two applications by fitting the NOLL-HL model and some famous models. The Cramérvon Mises $\left(\mathrm{W}^{*}\right)$, Anderson-Darling $\left(\mathrm{A}^{*}\right)$, Akaike information criterion (AIC) and the Bayesian information criterion (BIC) have been chosen for comparison of models for the first two examples.

The exponentiated half-logistic (EHL) distribution (Kang and Seo [14]), Kumaraswamy Half-Logistic distribution (KwHL) (Cordeiro and de Castro [8]), The Beta Half-Logistic (BHL) (Jones [13]), McDonald Half-Logistic distributin (Oliveria et.al [18]), Gamma distribution (Ga) with shape parameter $\alpha$ and scale parameter $\beta$ and Weibull distribution (W) with cdf $F_{W}(x)=1-\mathrm{e}^{-\alpha \mathrm{x}^{\beta}}$ have been selected for comparison in two examples. The parameters of models have been estimated by the MLE method.

\subsection{Data set I}

The first data are the prices of the 31 different childrens wooden toys on sale in a Suffolk craft shop in April 1991 : 4.2, 1.12, 1.39, 2, 3.99, 2.15, 1.74, 5.81, 1.7, 2.85, 0.5, 0.99, 11.5, 5.12, 0.9, 1.99, 6.24, 2.6, 3, 12.2, 7.36, 4.75, $11.59,8.69,9.8,1.85,1.99,1.35,10,0.65,1.45$. These data was analayzed by Shafiee et.al [20].

In the Table 1 , a summary of the fitted information criteria and estimated MLE's for this data with mentioned models, respectively. . As you see, the NOLL-HL is selected as the best model with more criteria. The histogram of the data set I and the plots of fitted pdf are displayed in Figure 7. 
Table 1. Result for data set I

\begin{tabular}{|c|c|c|c|c|c|c|}
\hline model & estimatte & parameters (se) & $W^{*}$ & $A^{*}$ & $A I C$ & $B I C$ \\
\hline NOLL-HL $(\alpha, \beta)$ & $\begin{array}{c}2.852 \\
(0.613)\end{array}$ & $\begin{array}{c}0.246 \\
(0.053)\end{array}$ & 0.030 & 0.231 & 148.05 & 150.91 \\
\hline OLLHL $(\alpha)$ & $\begin{array}{c}0.37 \\
(0.057)\end{array}$ & & 0.221 & 1.324 & 178.35 & 179.79 \\
\hline $\operatorname{EHL}(\alpha)$ & $\begin{array}{c}3.453 \\
(0.620)\end{array}$ & & 0.104 & 0.635 & 203.94 & 205.27 \\
\hline $\operatorname{KwHL}(\alpha, \beta)$ & $\begin{array}{l}1.453 \\
(0.510)\end{array}$ & $\begin{array}{l}0.298 \\
(0.059)\end{array}$ & 0.107 & 0.306 & 152.99 & 155.86 \\
\hline$\overline{\mathrm{BHL}}(\alpha, \beta)$ & $\begin{array}{c}1.313 \\
(0.396)\end{array}$ & $\begin{array}{c}0.297 \\
(0.060)\end{array}$ & 0.110 & 0.670 & 153.19 & 156.06 \\
\hline $\operatorname{McHL}(\alpha, \beta, \gamma)$ & $\begin{array}{c}0.124 \\
(0.078)\end{array}$ & $\begin{array}{cc}0.219 & 10.588 \\
(0.063) & (13.372)\end{array}$ & 0.044 & 0.302 & 155.59 & 161.32 \\
\hline $\mathrm{W}(\alpha, \beta)$ & $\begin{array}{c}0.155 \\
(0.057)\end{array}$ & $\begin{array}{c}1.228 \\
(0.169)\end{array}$ & 0.127 & 0.767 & 153.57 & 156.44 \\
\hline $\mathrm{Ga}(\alpha, \beta)$ & $\begin{array}{c}1.487 \\
(0.343)\end{array}$ & $\begin{array}{c}2.851 \\
(0.781)\end{array}$ & 0.114 & 0.692 & 152.91 & 155.78 \\
\hline
\end{tabular}

Figure 7. Histogram and estimated pdf for data set $I$.
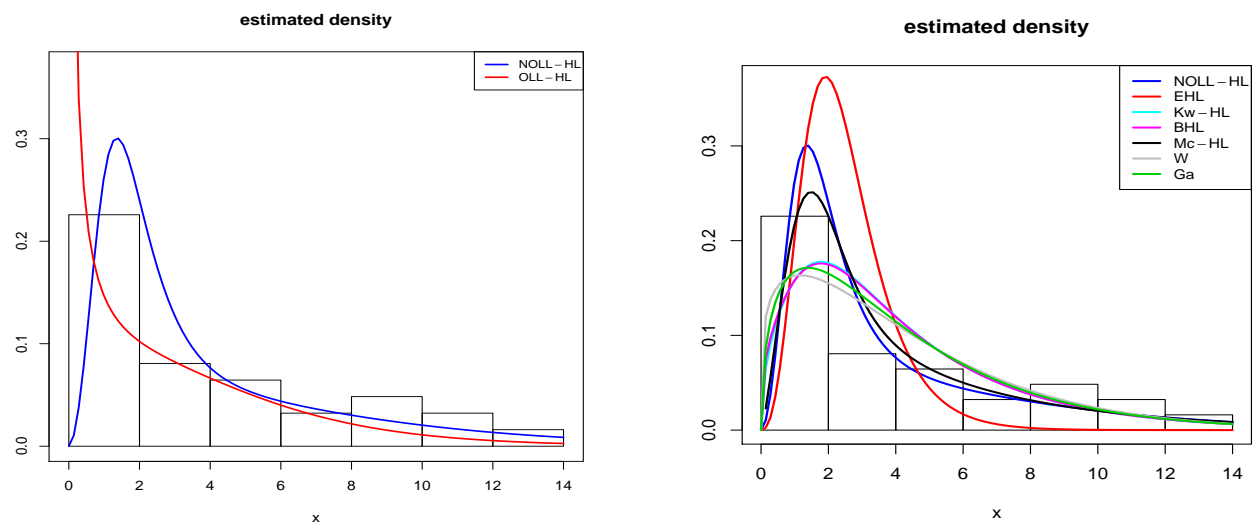

\subsection{Data set II}

The second real data set is related to failure time of 50 componnets (unit: 1000 hours) were reported by Murthy [17] (p180) . The data are: $0.036,0.058,0.061,0.074,0.078,0.086,0.102,0.103,0.114,0.116,0.148,0.183,0.192$, $0.254,0.262,0.379,0.381,0.538,0.570,0.574,0.590,0.618,0.645,0.961,1.228,1.600,2.006,2.054,2.804$, $3.058,3.076,3.147,3.625,3.704,3.931,4.073,4.393,4.534,4.893,6.274,6.816,7.896,7.904,8.022,9.337$, $10.94,11.02,13.88,14.73,15.08$.

Similar to the previous application example, we have Tables 2 . As it is clear, the NOLL-HL is selected as the best model with more criteria. The histogram of the data set II and the plots of fitted pdf are displayed in Figure 8 . 
Table 2. Result for data set II

\begin{tabular}{|c|c|c|c|c|c|c|c|}
\hline model & estimatte & paramete & rs (se) & $W^{*}$ & $A^{*}$ & $A I C$ & $B I C$ \\
\hline NOLL-HL $(\alpha, \beta)$ & $\begin{array}{c}0.690 \\
(0.112)\end{array}$ & $\begin{array}{c}0.245 \\
(0.043)\end{array}$ & & 0.103 & 0.690 & 204.61 & 208.44 \\
\hline OLLHL $(\alpha)$ & $\begin{array}{c}0.364 \\
(0.043)\end{array}$ & & & 0.214 & 1.406 & 218.38 & 220.29 \\
\hline $\operatorname{EHL}(\alpha)$ & $\begin{array}{c}0.867 \\
(0.122)\end{array}$ & & & 0.204 & 1.308 & 323.97 & 325.88 \\
\hline $\mathrm{KwHL}(\alpha, \beta)$ & $\begin{array}{c}0.337 \\
(0.097)\end{array}$ & $\begin{array}{c}0.266 \\
(0.041)\end{array}$ & & 0.206 & 1.329 & 216.67 & 220.50 \\
\hline$\overline{\mathrm{BHL}}(\alpha, \beta)$ & $\begin{array}{c}0.405 \\
(0.078)\end{array}$ & $\begin{array}{c}0.245 \\
(0.040)\end{array}$ & & 0.183 & 1.188 & 213.18 & 217.01 \\
\hline $\operatorname{McHL}(\alpha, \beta, \gamma)$ & $\begin{array}{c}0.127 \\
(0.057)\end{array}$ & $\begin{array}{c}0.196 \\
(0.042)\end{array}$ & $\begin{array}{c}4.314 \\
(2.207) \\
\end{array}$ & 0.134 & 0.866 & 209.75 & 215.49 \\
\hline $\mathrm{W}(\alpha, \beta)$ & $\begin{array}{c}0.541 \\
(0.099)\end{array}$ & $\begin{array}{c}0.661 \\
(0.074)\end{array}$ & & 0.152 & 0.954 & 208.72 & 212.55 \\
\hline $\mathrm{Ga}(\alpha, \beta)$ & $\begin{array}{c}0.545 \\
(0.090)\end{array}$ & $\begin{array}{c}6.127 \\
(1.555)\end{array}$ & & 0.148 & 0.948 & 208.87 & 212.69 \\
\hline
\end{tabular}

Figure 8. Histogram and estimated pdf for data set II.
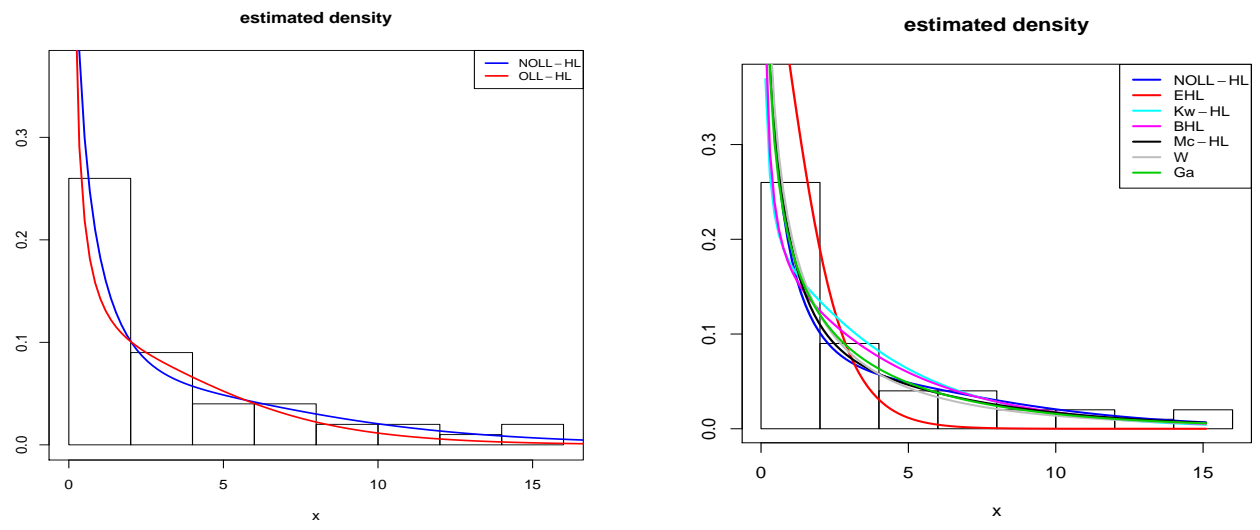

\section{Conclusions}

We introduce a new two-parameter extension of half-logiostic distributions called the new odd log-logistic halflogistic (NOLL-HL) family. Some characteristics of the new family, such as moments, mean past lifetime, coefficients of skewness and kurtosis, entropy, order statistics and asymptotic properties are obtained. We estimate the parameters using maximum likelihood and other different methods. The Bias and MSE plots of parameters for all methods, will approach to zero with the increase in the volume of the sample which verifies the validity of the these estimation methods. The flexibility of this distribution is assessed by applying it to real data sets and comparing purpose distribution with others. The results of tables and figures illustrate the new models provide consistently better fits than other competitive models for these data sets. So Applications demonstrate the importance of the new family. 


\section{Appendix A}

The element of information matrix are given by

$$
\begin{gathered}
U_{\alpha \alpha}=-\sum_{i=1}^{n}\left[\frac{1-q_{i}}{\alpha+(\beta-\alpha) q_{i}}\right]^{2}-2 \sum_{i=1}^{n} \frac{q_{i}^{\alpha}\left(1-q_{i}\right)^{\beta}\left[\log \left(q_{i}\right)\right]^{2}}{\left[q_{i}^{\alpha}+\left(1-q_{i}\right)^{\beta}\right]^{2}} \\
U_{\beta \beta}=-\sum_{i=1}^{n}\left[\frac{q_{i}}{\alpha+(\beta-\alpha) q_{i}}\right]^{2}-2 \sum_{i=1}^{n} \frac{q_{i}^{\alpha}\left(1-q_{i}\right)^{\beta}\left[\log \left(1-q_{i}\right)\right]^{2}}{\left[q_{i}^{\alpha}+\left(1-q_{i}\right)^{\beta}\right]^{2}} \\
U_{\alpha \beta}=-\sum_{i=1}^{n} \frac{q_{i}\left(1-q_{i}\right)}{\left[\alpha+(\beta-\alpha) q_{i}\right]^{2}}+2 \sum_{i=1}^{n} \frac{q_{i}^{\alpha}\left(1-q_{i}\right)^{\beta} \log \left(q_{i}\right) \log \left(1-q_{i}\right)}{\left[q_{i}^{\alpha}+\left(1-q_{i}\right)^{\beta}\right]^{2}}
\end{gathered}
$$

\section{Appendix B: Four useful power series}

We present four power series required for the algebraic developments in Section 2.

First, for $a>0$ real non-integer, we have the binomial expansion

$$
(1-u)^{a}=\sum_{j=0}^{\infty}(-1)^{j}\left(\begin{array}{l}
a \\
j
\end{array}\right) u^{j},
$$

and second

$$
\begin{gathered}
u^{a}=\sum_{j=0}^{\infty} s_{j}(a) u^{j}, \\
(1-u)^{-a}=\sum_{j=0}^{\infty} \frac{\Gamma(a+j)}{j ! \Gamma(a)} u^{j}
\end{gathered}
$$

where $s_{k}(a)=\sum_{j=k}^{\infty}(-1)^{j+k}\left(\begin{array}{l}a \\ j\end{array}\right)\left(\begin{array}{l}j \\ k\end{array}\right)$ the binomial coefficient is defined for any real.

Third, expanding $z^{\lambda}$ in Taylor series, we can write

$$
\begin{aligned}
& z^{\lambda}=\sum_{k=0}^{\infty}(\lambda)_{k}(z-1)^{k} / k !=\sum_{i=0}^{\infty} f_{i} z^{i} \\
& f_{i}=f_{i}(\lambda)=\sum_{k=i}^{\infty} \frac{(-1)^{k-i}}{k !}\left(\begin{array}{c}
k \\
i
\end{array}\right)(\lambda)_{k}
\end{aligned}
$$

and $(\lambda)_{k}=\lambda(\lambda-1) \ldots(\lambda-k+1)$ denotes the descending factorial.

Fourth we obtain an expansion for $\left[u^{\alpha}+(1-u)^{\beta}\right]^{c}$ where $0<u<1$. We can write

$$
\left[u^{\alpha}+(1-u)^{\beta}\right]=\sum_{j=0}^{\infty} t_{j} u^{j},
$$

where $t_{j}=t_{j}(\alpha, \beta)=\sum_{i=j}^{\infty}(-1)^{i+j}\left(\begin{array}{c}\alpha \\ i\end{array}\right)\left(\begin{array}{l}i \\ j\end{array}\right)+(-1)^{j}\left(\begin{array}{l}\beta \\ j\end{array}\right)$. Then, using (34), we have

$$
\left[u^{\alpha}+(1-u)^{\beta}\right]^{c}=\sum_{i=0}^{\infty} f_{i}\left(\sum_{j=0}^{\infty} t_{j} u^{j}\right)^{i},
$$


where $f_{i}=f_{i}(c)$. Finally, using again equations (35) and (32), we have

$$
\left[u^{\alpha}+(1-u)^{\beta}\right]^{c}=\sum_{j=0}^{\infty} h_{j}(\alpha, \beta ; c) u^{j},
$$

where $h_{j}(\alpha, \beta ;, c)=\sum_{i=0}^{\infty} f_{i} m_{i, j}$ and (for $\left.i \geq 0\right) m_{i, j}=\left(j t_{0}\right)^{-1} \sum_{m=1}^{j}[m(j+1)-j] t_{m} m_{i, j-m}($ for $j \geq 1)$ and $m_{i, 0}=t_{0}^{i}$.

\section{Conclusion}

We introduce a new two-parameter extension of half-logiostic distributions called the new odd log-logistic halflogistic (NOLL-HL) family. Some characteristics of the new family, such as moments, mean past lifetime, coefficients of skewness and kurtosis, entropy, order statistics and asymptotic properties are obtained. We estimate the parameters using maximum likelihood and other different methods. The Bias and MSE plots of parameters for all methods, will approach to zero with the increase in the volume of the sample which verifies the validity of the these estimation methods. The flexibility of this distribution is assessed by applying it to real data sets and comparing purpose distribution with others. The results of tables and figures illustrate the new models provide consistently better fits than other competitive models for these data sets. So Applications demonstrate the importance of the new family.

\section{Acknowledgement}

The authors would like to thank two anonymous referees and the associate editor for their helpful comments that have considerably improved this manuscript.

\section{REFERENCES}

1. T. W. Anderson, and D. A. Darling, Asymptotic theory of certain" goodness of fit" criteria based on stochastic processes , The annals of mathematical statistics, pp. 193-212, 1952.

2. Alizadeh, M., MirMostafee, S. M. T. K., Ortega, E. M., Ramires, T. G., and Cordeiro, G. M. The odd log-logistic logarithmic generated family of distributions with applications in different areas Journal of Statistical Computation and Simulation, vol. 4(1), 6, 2017.

3. A. Alzaatreh, C. Lee, and F. Famoye, A new method for generating families of continuous distributions, Metron, vol. 71(1), pp. 63-79, 2013.

4. N. Balakrishnan, Order statistics from the half logistic distribution, Journal of Statistical Computation and Simulation, vol. 20(4), pp. 287-309, 1985 .

5. R. C. H. Cheng, N. A. K. Amin, Maximum product-of-spacings estimation with applications to the lognormal distribution, Technical Report, Department of Mathematics, University of Wales, 1979.

6. R. C. H. Cheng, N. A. K. Amin, Estimating parameters in continuous univariate distributions with a shifted origin, Journal Of The Royal Statistical Society Series B, vol. 3, pp. 394-403, 1983.

7. K. Choi, and W. Bulgren, An estimation procedure for mixtures of distributions, Journal of the Royal Statistical Society. Series B, pp. 444-460, 1968.

8. G. M. Cordeiro, and M. de Castro, A new family of Generalized distributions, Journal of Statistical Computation and Simulation, vol. 81, pp. 883-898, 2011.

9. Cordeiro, G. M., Alizadeh, M., Ozel, G., Hosseini, B., Ortega, E. M. M., and Altun, E. The generalized odd log-logistic family of distributions: properties, regression models and applications Journal of Statistical Computation and Simulation, vol. 87(5), pp. 908-932, 2017.

10. S. Dey, J. Mazucheli and S. Nadarajah, Kumaraswamy distribution: different methods of estimation, Computational and Applied Mathematics, pp. 1-18, 2017.

11. J. U. Gleaton, and J. D. Lynch, Properties of generalized log-logistic families of lifetime distributions, Journal of Probability and Statistical Science, vol. 4(1), pp. 51-64, 2006

12. I. S. Gradshteyn, and I. M. Ryzhik, Table of Integrals, Series, and Products, sixth edition, Academic Press, New York, 2007.

13. M. C. Jones, Families of distributions arising from distributions of order statistics, Test, vol. 13(1), pp. 1-43, 2004.

14. S. B. Kang, and J. I. Seo, Estimation in an exponentiated half logistic distribution under progressively type-II censoring, Communications for Statistical Applications and Methods, vol. 18(5), pp. 657-666, 2011. 
15. Leadbetter, M. R., Lindgren, G., and Rootzn, H, Extremes and related properties of random sequences and processes, pringer Science and Business Media, 2012.

16. A. W. Marshall, and I. Olkin, A new method for adding a parameter to a family of distributions with application to the exponential and Weibull families, Biometrika, vol. 84, pp. 641-652, 1997.

17. D. P. Murthy, M. Xie, and R. Jiang, Weibull models, John Wiley and Sons, Vol. 505, 2004.

18. J. Oliveira, J. Santos, C. Xavier, D. Trindade, and G. M. Cordeiro, The McDonald half-logistic distribution: Theory and practice, Communications in Statistics-Theory and Methods, vol. 45(7), pp. 2005-2022, 2016.

19. A. Rényi, On measures of entropy and information, Proceedings of the Fourth Berkeley Symposium on Mathematical Statistics and Probability, vol. 1, pp. 547-561, 1961.

20. S. Shafiei, S. Darijani, and H. Saboori, Inverse Weibull power series distributions: properties and applications, Journal of Statistical Computation and Simulation, vol. 86(6), pp. 1069-1094, 2016.

21. C. E. Shannon, Prediction and entropy of printed English, The Bell System Technical Journal, vol. 30, pp. 50-64, 1951.

22. E. W. Stacy, A generalization of the gamma distribution, The Annals of mathematical statistics, vol. 30, pp. 1187-1192, 1962.

23. J. J. Swain, S. Venkatraman, and J. R. Wilson, Least-squares estimation of distribution functions in johnson's translation system, Journal of Statistical Computation and Simulation, vol. 29(4), pp. 271-297, 1988. 